\title{
From the hypertransaminasemia symptoms to the recognition of late-onset Pompe disease in a 12-year-old boy
}

\author{
Ewa Grzywna, Jarosław Kwiecień \\ Chair and Department of Paediatrics in Zabrze, Medical University of Silesia in Katowice, Poland
}

ABSTRACT

The paper presents the case of a 12-year-old boy hospitalised due to persistent hypertransaminasemia of unknown origin, in whom rare metabolic disease - Pompe disease, was finally diagnosed. We discuss the possible symptoms and the diagnostic criteria for Pompe disease, as well as modern genetic methods of diagnosing. The importance of including this metabolic disease in differential diagnosis of hypertransaminasemia was underlined. The recombinant human a-glucosidase as the enzyme replacement therapy makes nowadays the early diagnosis of Pompe disease especially important.

KEY WORDS:

metabolic disorders, Pompe disease, hypertransaminasemia.

\section{INTRODUCTION}

Pompe disease is a rare, autosomal recessive disorder of glycogen storage. The disease affects approximately $1 / 40,000$ in live births, although screening studies demonstrate it may be higher [1-3]. The disease is caused by the mutation of GAA gene, located on 17q25 chromosome, encoding the enzyme acid $\alpha 1,4$-glucosidase, which intracellularly breaks down glycogen. The first symptoms of the disease appear from infancy to adulthood and depend on the degree of lysosomal a1,4-glucosidase activity deficiency. In the typical infantile-onset form of the disorder, this activity is $<1 \%$ and progressive weakness and generalized hypotonia, macroglossia and increasing hypertrophic cardiomyopathy are observed in these patients $[1,2]$. In late-onset Pompe disease, the main problem is progressive weakness of skeletal muscles and increasing respiratory disorders $[1,2]$.

\section{CASE REPORT}

The aim of the study is to present the case of a 12-yearold boy hospitalised for due to persistent hypertrans- aminasemia, in whom Pompe disease was finally diagnosed.

Family, pregnancy and delivery history of the patient was unremarkable. The developmental delay was observed but only in terms of motor development; he walked independently only at 18 months of age. The social activity, speech and intellectual development of the child were normal. At 8 years of age, in routine tests elevated liver enzymes were found, which repeated in follow-up examinations (up to Alat-171 U/1, Ast-136 $\mathrm{U} / \mathrm{l}$ ) and was present for over 6 months. The clinical diagnosis performed at that time excluded $\alpha 1$-antitrypsin deficiency, autoimmune hepatitis, Wilson's disease and the most common infections with hepatotropic viruses. Because of unexplained chronic hypertransaminasemia, liver biopsy was also performed, revealing features of parenchymal lesions with minimal inflammatory activity.

Among many parameters assessed for diagnosis of hypertransaminasemia, the creatine kinase (CK) was also performed at that time, revealing increased activity of CK-1136 U/l. For this reason the child was additionally consulted by the specialist in neurology, but no sings

\section{ADDRESS FOR CORRESPONDENCE:}

Jarosław Kwiecień, Chair and Department of Paediatrics in Zabrze, Medical University of Silesia in Katowice, Poland, e-mail: jkwiecien@sum.edu.pl 

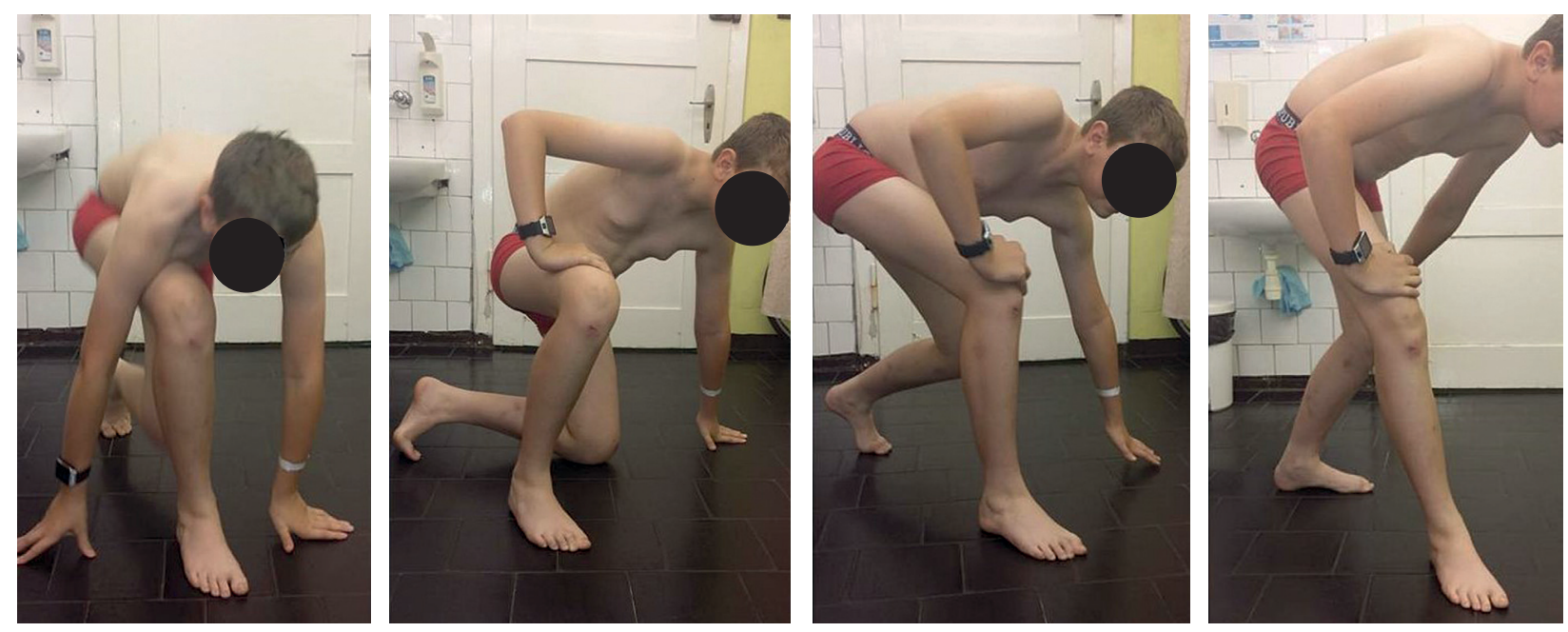

FIGURE 1. Gowers sign - patient "climb up" his thighs with the aid of hands while standing up

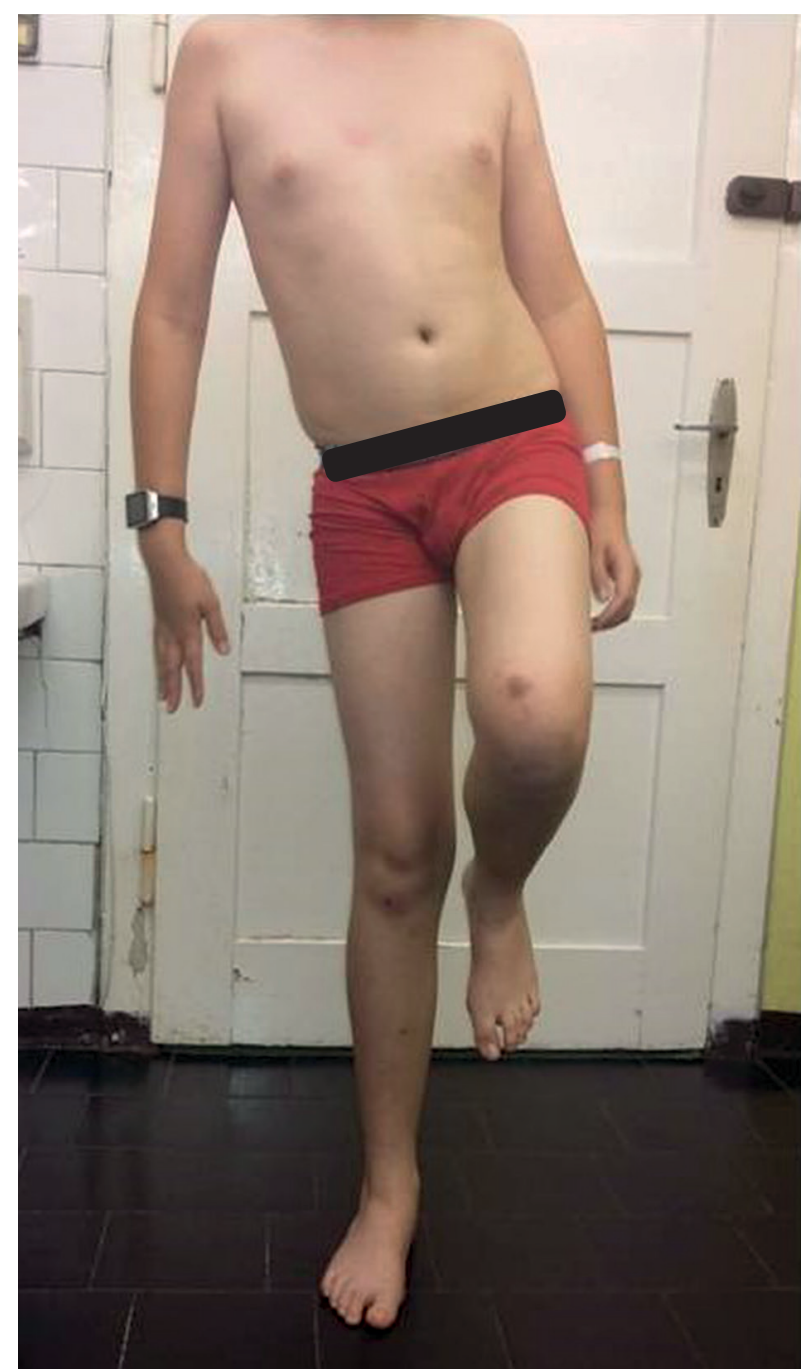

FIGURE 2. Duchenne sign - shifting the upper body towards the stance leg

of neuromuscular disorder were found. The neurologist recommended periodic assessment of CK and reconsultation. Unfortunately, the scheduled gastrological and neurological control visits were irregular and without CK activity assessment.
On his second admission to the gastroenterology department at the age of 12 , elevated values of alanine transaminase, aspartate transaminase, lactate dehydrogenase and creatine kinase were found, with increase both in AlAT, as well as in CK activity (Alat-249 U/l, Ast-213 U/l, LDH-754 U/l, CK-2020 U/1). The abdominal ultrasound, ECG, echocardiography and spirometry examinations showed no deviations. Due to the postural defect, proximal muscle weakness, absent knee and ankle reflexes, and the positive Gowers sign (Figure 1) and Duchenne sign (Figure 2) which were found in the neurological examination, the diagnostic tests were extended to include the determination of alpha-glucosidase activity, showing its decrease in the dried blood spot test, followed by the study of peripheral blood leukocytes. The results of a-glucosidase activity were 0,05 (normal values 0.29-0.49) and respectively 0.07 (normal values $0.31-0.52$ ), suggesting the diagnosis of Pompe disease (the assessment made on request of the hospital in the Laboratory of Psychiatry and Neurology Institute in Warsaw).

Thereafter, the diagnosis of Pompe disease was confirmed by molecular examination of GAA gene, showing the presence of heterozygous c.307T $>$ G and c.32-13T $>\mathrm{G}$ mutations. The boy was qualified for enzyme replacement therapy under the drug program.

\section{DISCUSSION, SYMPTOMS AND POSSIBLE TREATMENT}

In late-onset Pompe disease, the first symptoms may include increased fatigue and muscle pain. Gradually paresis of the pelvic girdle and shoulder girdle increases, causing difficulties in getting up, climbing stairs and raising the arms, among others. An additional indication may be increased values of creatine kinase, often also increased values of alanine transaminase and aspartate transaminase. As the disease progresses, the respiratory muscles, including the diaphragm, are affected, leading to respiratory failure. Patients develop sleep apnea, 
headaches may occur secondary to hypoxia. Although in the late form the dominant symptoms are muscular, Pompe disease is a multi-system disorder, and the enzyme defect affects other tissues and organs as well. Patients may suffer from vascular (including cerebral) disorders, peripheral and central nervous system disorders, hearing loss, scoliosis, bone mass loss, as well as disorders of the digestive or urinary system $[1,2,4]$. The absence of tendon reflexes from the lower limbs in our patient indicates the coexistence of motor neuropathy with the symptoms of myopathy [5].

The diagnosis of the disease is based on a documented deficit in alpha glucosidase activity. It can be determined by the dried blood spot test, in peripheral blood leukocytes or in skin fibroblasts. For confirmation, molecular tests are performed [2]. The a-glucosidase gene is located on the 17q25.2-q25.3 chromosome. To date, over 500 mutations associated with Pompe disease have been described. The mutation c.307T $>$ G found in the presented child is a missense mutation, while c.32-13T $>\mathrm{G}$ is a mutation leading to impaired exon 2 splicing and reducing the expression of the alpha glucosidase gene $[2,6,7]$. Unlike others, the c.32-13T> G mutation is quite common in patients of Caucasian origin diagnosed with Pompe disease, and the frequency of this allele in this group is $40 \%$ to $70 \%$ [7].

Currently, enzyme replacement therapy is available for the treatment of this disease, and the approved drug is alglucosidase a (recombinant human $\alpha$-glucosidase). It is administered to patients every 2 weeks as an intravenous infusion and, according to studies, it slows disease progression, although in most patients it does not cause significant disease regression $[1,2,8]$. One of the reasons for the poorer drug response in skeletal muscle may be the low abundance of the cation-independent mannose-6-phosphate receptor (CI-MPR) that mediates receptor-mediated uptake of recombinant human alpha-glucosidase. The expression of CI-MPR may be influenced by adjuvant therapy with $\beta 2$-agonists [9]. Currently, next-generation enzyme replacement therapies are in the clinical trial phase, and gene therapy research is also underway $[1,2,8]$.

In the diagnosis of hypertransaminasemia, it is appropriate to determine the concentration of creatine kinase. Moreover, taking into account the natural clinical course as well as the available therapy, diagnostics for this rare disease in patients with elevated creatine kinase concentration should be considered. There is to underline, that in more and more rare diseases we have possibility to successfully treat the child and stop the development of the disease. For this reason, early diagnosis of genetic disorders is very important. There is also to keep in mind that we have available tools for diagnosing Pompe disease, starting with simple CK assessment, up to a-glucosidase activity using the dried blood spot test.

\section{DISCLOSURE}

The authors declare no conflict of interest.

\section{REFERENCES}

1. Meena NK, Raben N. Pompe Disease: New Developments in an Old Lysosomal Storage Disorder. Biomolecules 2020; 10: 1339.

2. Taverna S, Cammarata G, Colomba P, et al. Pompe disease: pathogenesis, molecular genetics and diagnosis. Aging (Albany NY) 2020; 12: 15856-15874.

3. Sawada T, Kido J, Nakamura K. Newborn Screening for Pompe Disease. Int J Neonatal Screen 2020; 6: 31.

4. Filosto M, Todeschini A, Cotelli MS, et al. Non-muscle involvement in late-onset glycogenosis II. Acta Myol 2013; 32: 91-94.

5. Tsai LK, Hwu WL, Lee NC, et al. Clinical features of Pompe disease with motor neuronopathy. Neuromuscul Disord 2019; 29: 903-906.

6. Herzog A, Hartung R, Reuser AJ, et al. A cross-sectional single-centre study on the spectrum of Pompe disease, German patients: molecular analysis of the GAA gene, manifestation and genotype-phenotype correlations. Orphanet J Rare Dis 2012; 7: 35.

7. Peruzzo P, Pavan E, Dardis A. Molecular genetics of Pompe disease: a comprehensive overview. Ann Transl Med 2019; 7: 278.

8. Do HV, Khanna R, Gotschall R. Challenges in treating Pompe disease: an industry perspective. Ann Transl Med 2019; 7: 291.

9. Koeberl DD, Li S, Dai J, et al. $\beta 2$ Agonists enhance the efficacy of simultaneous enzyme replacement therapy in murine Pompe disease. Mol Genet Metab 2012; 105: 221-227. 